\title{
Predictors of Sleep Recall Bias in College Students
}

\author{
Pranjal Srivastava, Mina Takegami \\ Mentor: Dr. Ching-Hua Chen
}

\begin{abstract}
College students are known to have unhealthy levels of sleep. Using data from the StudentLife Dataset, we analyzed the predictors of "recall bias" regarding sleep. We compared the average of a running, daily, self-reported sleep value over a period of time to the average sleep amount perceived by the students in their PSQI (Pittsburgh Sleep Quality Index) tests. We found that the variability in the measure of self-assessment of health was related to errors in sleep recall, as were some individual parameters.
\end{abstract}

\section{Introduction}

Sleep is an important indicator and factor for human health [1]. Deprivation of sleep among certain groups, like college students, is a serious problem. In particular, for students, sleep has been shown to affect grades and health [7]. Some recent work has suggested more than a third of the college community perceives itself to be sleep deprived [9]. As shown in [3], the relationship can go the other way too -- varied parameters like gender, grades and year (freshman, junior etc.) can affect sleep.

Perception of sleep is as important almost as important as sleep itself. In diseases like Insomnia, for example, the underestimation of sleep by the patients is problematic and may cause them to overestimate their problems [1]. The perception of how much we sleep very much affects how much sleep we will get in the future. People who overestimate or underestimate the amount of sleep they get are at a risk of making decisions regarding their future sleep schedules based on their incorrect perception of their sleep history.

Connected to the sleep deprivation issue is the idea of "recall bias" -- defined as an error in recollection of a certain parameter by the subject after a period of time. Whether underreporting or overreporting, the effect of the bias is to increase the the difference between real and reported values [6]. A lack of rigidity regarding sleep schedules (different timings and hours of sleep every night) may increase the recall bias, which was among one of the different parameters we studied to explain recall bias by college students regarding sleep.

\section{Problem Statement}

There is a difference between self-reported recollections of sleep over a long period of time and daily reported sleep amounts over the same time period. This phenomenon is referred to as "recall bias". Our objective is to predict the degree of recall bias based on certain personality / behavioural 
features. In our study, we represent recall bias by the difference between the recalled sleep averages and the sleep average computed from daily reports.

The contributions of our work are as follows:

- Enables a study of recall bias in students.

- Finds parameters that can help finding students that may underestimate or overestimate sleep.

Outline of Paper

The remainder of this paper is organized as followed: We first discuss our methods used, followed by our results. We conclude with a discussion of findings and future work.

\section{Methods and Approach}

\section{The StudentLife Dataset}

The StudentLife Dataset [10] contains partial or complete sensor data, EMA data, survey responses and educational data for 60 students at Dartmouth University over the 10 week spring term. We focused only on using the survey responses (for example sensor data like time phone used and survey responses). Some of the surveys the student took over the period were administered both before and after the period in which sleep data was collected, and will be called Pre and Post surveys, respectively.

\section{Procedure}

To research the predictors of "recall bias" regarding sleep, we used the StudentLife Dataset from Dartmouth University, comparing the average of a running, daily, self - reported sleep value over a period of time to the average sleep amount perception of people, as reported in their PSQI tests (Pittsburgh Sleep Quality Index). Some of these responses were recorded as ranges; in such cases, we assumed that the meanof the two endpoints of the range as the recalled sleep duration.

Of the 60 people included in the dataset, 40 students were randomly selected to be train a Random Forest regression model.

Of these 40 students, only 26 had complete data that could be used for working out correlations between the calculated sleep error value \& possible predictors, like respect, piazza usage, standard deviation of the daily data (a proxy for measuring variability of sleep schedule) and relative importance (using RandomForest). The training data was of two types, one in which all students with usable, reported values of SleepError and other parameters (See Appendix) were used (25 students in total, 1 had to be excluded due to incomplete data) and the other in which only students who had reported more than 20 days for the running average of sleep were used (18 
students in total, 1 excluded due to incomplete data, 7 excluded since they did not fulfill the constraints, such as at least 10 responses collected for daily sleep reporting).

These datasets both had 72 potential variables collected from other surveys in the StudentLife Dataset and statistical analyses of various aspects of the surveys including standard deviation and averages, and differences between pre and post surveys of various attributes. These 72 variables were then tested to see how well they could predict the variability in the SleepError. The "best" variables were considered to be the ones that had the highest \%IncMSE (a randomForest criteria) during the relative importance analysis, and various combinations of one, two and three variables were tried to find the model that produced the highest \%Variation Explained.

Then, by using the predict() function in R, the model was used to extrapolate values for SleepError based on the chosen variables and data type. Then, the root mean square of the difference between the actual sleep error and the predicted one was calculated with an online tool, and the best model was the one that had the least RMSE. The results from all the models can be seen in supplemental material. ${ }^{1}$

\section{Evaluation}

72 variables were tested (Check Appendix) in the dataset. At first, only individuals which had incomplete data were excluded from the analysis. The variable named SocialStDevO, which was defined as the standard deviations of the Post Flourishing Scale survey, was among the best predictors of Sleep Error in all variables tested.

When the dataset was restricted to only students who had self-reported more than 20 days of sleep values, the variable GoodO, defined as their response to the question "I am a good person and live a good life" on the Flourishing Scale on a range from 1-10 was found to be an appropriate predictor of Sleep Error. Together, these variables produced one of the best models, which where then used to predict the Sleep Error of the 10 students with complete data in the testing set. The resulting model had a RMSE of 0.6095338471 , which was significantly better than the other models (logarithmic regression, RandomForest Models with other parameters) which had an RMSE of around one or more.

These findings are interesting, because they show us that the variability in measures of self-assessment of health was related to errors in sleep recall, which no other previous study had shown.

1https://docs.google.com/spreadsheets/d/e/2PACX-1vRljPzCivwarlR1Wdl4HU5fZ6CUhufXIOftuyOrg l0fW9mMVf6gSU2SfldtPlamznm4Q G67ELX4I6x/pubhtml 


\section{Conclusion and Future Work}

Due to the very small size of the dataset, these results can not be considered final and further research is needed into the link between the Flourishing Scale test and Sleep Error, which appear related, especially with regard to the variance in a person's responses on the whole test and their responses to the question "I am a good person and live a good life". Other sources of error can be randomForest itself, which is not able to handle values that are not present in a dataset while computing a regression correctly. Error may also stem from the averages of running sleep values that were computed by both human and google sheets.

Further research can also be conducted into how Sleep Error is related to non-numeric data, which was not looked at in our study, perhaps using a classification in randomForest. 


\section{References}

1. Harvey, Allison $G$ and Nicole K Y Tang. "(Mis)perception of sleep in insomnia: a puzzle and a resolution" Psychological bulletin vol. 138,1 (2011): 77-101.

2. Lund, Hannah G., et al. "Sleep Patterns and Predictors of Disturbed Sleep in a Large Population of College Students.” Journal of Adolescent Health, vol. 46, no. 2, Feb. 2010, pp. 124-132., doi:10.1016/j.jadohealth.2009.06.016.

3. Tsai, Ling-Ling, and Sheng-Ping Li. "Sleep Patterns in College Students." Journal of Psychosomatic Research, vol. 56, no. 2, Feb. 2004, pp. 231-237., doi:10.1016/s0022-3999(03)00507-5.

4. Walter C. Buboltz Jr PhD, Franklin Brown MA \& Barlow Soper PhD (2001) Sleep Habits and Patterns of College Students: A Preliminary Study, Journal of American College Health, 50:3, 131-135, DOI: 10.1080/07448480109596017

5. Pilcher, June J., et al. "Sleep Quality versus Sleep Quantity: Relationships between Sleep and Measures of Health, Well-Being and Sleepiness in College Students." Journal of Psychosomatic Research, vol. 42, no. 6, June 1997, pp. 583-596., doi:10.1016/s0022-3999(97)00004-4.

6. Raphael, Karen. "Recall Bias: A Proposal for Assessment and Control." International Journal of Epidemiology, vol. 16, no. 2, June 1987, pp. 167-170., doi:10.1093/ije/16.2.167.

7. Trockel, Mickey T., et al. "Health-Related Variables and Academic Performance Among First-Year College Students: Implications for Sleep and Other Behaviors." Journal of American College Health, vol. 49, no. 3, Mar. 2000, pp. 125-131., doi:10.1080/07448480009596294.

8. Caldwell, Karen, et al. "Developing Mindfulness in College Students Through Movement-Based Courses: Effects on Self-Regulatory Self-Efficacy, Mood, Stress, and Sleep Quality." Journal of American College Health, vol. 58, no. 5, 24 Oct. 2010, pp. 433-442., doi:10.1080/07448480903540481.

9. Moo-Estrella, Jesús, et al. "Evaluation of Depressive Symptoms and Sleep Alterations in College Students." Archives of Medical Research, vol. 36, no. 4, 2005, pp. 393-398., doi:10.1016/j.arcmed.2005.03.018.

10. Wang, Rui, Fanglin Chen, Zhenyu Chen, Tianxing Li, Gabriella Harari, Stefanie Tignor, Xia Zhou, Dror Ben-Zeev, and Andrew T. Campbell. "StudentLife: Assessing Mental Health, Academic Performance and Behavioral Trends of College Students using Smartphones." In Proceedings of the ACM Conference on Ubiquitous Computing. 2014. 
Appendix

\begin{tabular}{|c|c|c|c|}
\hline Parameter & Parameter & Parameter & Parameter \\
\hline StDev & Respect & HostileO & Guilty \\
\hline Days & Purposeful & EnthusiasticO & Scared \\
\hline PiazzaDays & Social & ProudO & Hostile \\
\hline PiazzaViews & Engaged & IrritableO & Enthusiastic \\
\hline PiazzaCont & Happy & AlertO & Proud \\
\hline PiazzaQues & Competent & InspiredO & Irritable \\
\hline PiazzaNote & Good & NervousO & Alert \\
\hline PiazzaAns & Optimist & DeterminedO & Inspired \\
\hline RespectO & SocialAvg & AttentiveO & Nervous \\
\hline PurposfulO & SocialStDev & JitteryO & Determined \\
\hline SocialO & DiffAvgFlo & ActiveO & Attentive \\
\hline EngagedO & DiffStDevFlo & AfraidO & Jittery \\
\hline HappyO & InterestedO & AvgPANASO & Active \\
\hline CompetentO & DistressedO & StDevPANASO & Afraid \\
\hline GoodO & UpsetO & Interested & AvgPANAS \\
\hline OptimistO & StrongO & Distressed & StDevPANAS \\
\hline SocialAvgO & GuiltyO & Upset & DiffAvgPANAS \\
\hline SocialStDevO & ScaredO & Strong & $\begin{array}{c}\text { DiffStDevPAN } \\
\text { AS }\end{array}$ \\
\hline
\end{tabular}

